\title{
NUMERICAL STUDY OF THE CREEP BUCKLING RESPONSE OF LAMINATED ORTHOTROPIC LINEAR VISCOELASTIC CYLINDRICAL SHELLS
}

\author{
Melanie Todt ${ }^{1 *}$, Rainer Tomas ${ }^{2}$, Thomas Koch ${ }^{2}$, and Heinz E. Pettermann ${ }^{1}$ \\ ${ }^{1}$ Institute of Lightweight Design and Structural Biomechanics, TU Wien, Austria \\ ${ }^{2}$ Mück Kunststofftechnik GmbH, Götzendorf a.d. Leitha, Austria \\ ${ }^{3}$ Institute of Materials Science and Technology, TU Wien, Austria \\ *melanie.todt@tuwien.ac.at
}

Creep buckling of laminated cylindrical shells made of glass fiber reinforced polyester resin is studied by means of the finite element method. The material behavior of the individual plies is modeled using the anisotropic linear-viscoelastic constitutive law presented in [1]. The input parameters for the constitutive law are derived using a periodic unit cell approach. The viscoelastic response of the matrix is determined by dynamic mechanical analysis experiments and the linear elastic properties of the fibers are taken from literature.

Various layups of the laminate as well as different loading scenarios such as pure axial loading or a pure lateral pressure are considered. The creep deformations as well as the time until buckling are evaluated for different load magnitudes with respect to the instantaneous buckling load, as illustrated in Figure 1 for the case of axial loading. The obtained results show a distinct impact of the lay-up on the creep buckling response and can serve as basis for the design of structures with high creep buckling resistance.
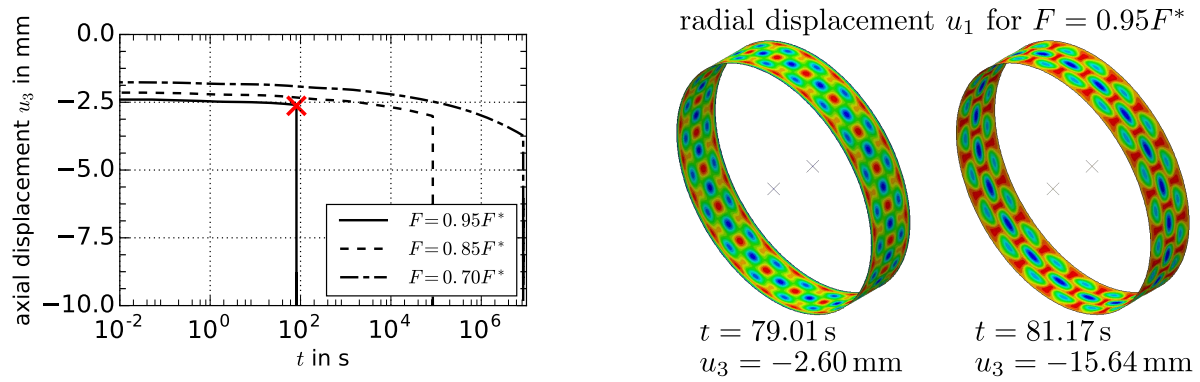

Figure 1: Creep response of a cylindrical shell with a $[0]_{4}$ layup subjected to purely axial loading and for different load magnitudes with respect to the instantaneous buckling load $F^{*}$. The onset of creep buckling is marked by the red cross and the contour plots show the radial displacements prior and during the collapse of the cylinder.

\section{Acknowledgments}

The funding by the Polymer Competence Center Leoben GmbH (PCCL) within the COMETprogram by the Austrian Federal Ministry for Transport, Innovation, and Technology, the Austrian Federal Ministry of Digital and Economic Affairs, Österreichische Forschungsförderungsgesellschaft mbH (FFG), the Provinces of Styria, Lower Austria, and Upper Austria, is gratefully acknowledged.

\section{References}

[1] H.E. Pettermann and A. DeSimone (2018) An anisotropic linear thermo-viscoelastic constitutive law. Mechanics of Time-Dependent Materials, 22, 421-433. 